\title{
Ionization Quantum Yields and Absorption Coefficients of Selected Compounds at 58.4 and $73.6-74.4 \mathrm{~nm}^{*}$
}

\author{
R. E. Rebbert and P. Ausloos \\ Institute for Materials Research, National Bureau of Standards, Washington, D.C. 20234
}

(April 15, 1971)

\begin{abstract}
The ionization quantum yields and the extinction coefficients of a number of compounds have been determined at the wavelengths of the helium $(58.4 \mathrm{~nm})$ and neon $(73.6-74.4 \mathrm{~nm})$ resonance lamps. These are lamps with thin aluminum windows $(100-200 \mathrm{~nm})$ which we inserted in a glass cell backed by a second cell. Both cells are provided with parallel plate electrodes and separated from each other by an aluminum window. The ionization quantum yields are based on ionization efficiency of argon which is unity. Hydrogen, which has an ionization quantum yield of 0.94 and 1.00 at $73.6^{-}$ 74.4 and $58.4 \mathrm{~nm}$ respectively, was used as a secondary standard because it yielded better defined saturation ion current plateaus. The extinction coefficients were determined in both a double cell and a single cell arrangement. The agreement between the two measurements was excellent. In general an inert diluent was added to the gas of interest in order to improve the plateau of the saturation ion current. These results are compared with the literature values, which were mainly determined in windowless systems with monochromators.
\end{abstract}

Key words: Absorption coefficient; helium resonance radiation; hydrocarbons: inorwanic molecules; ionization efficiency; ionization quantum yield; neon resonance radiation.

\section{Introduction}

In the past, photoionization quantum yields and absorption coefficients in the range 10 to $100 \mathrm{~nm}$ have been measured in windowless systems utilizing differential pumping techniques with monochromators. This method suffers because the path lengths are not well defined, which may explain why the agreement between different investigators is not always satisfactory [1] ${ }^{1}$. Recently de Reilhac et al., [2] have improved the measurement of absorption coefficients in the range 10 to $50 \mathrm{~nm}$ using absorption cells with aluminum windows, which provide a well defined path length. However, so far not many measurements have been made.

In the course of our study [3] of the vacuum ultraviolet photolysis of various organic compounds with rare gas resonance lamps, it became necessary for us to know very exactly the ionization quantum yields and the absorption coefficients of the different gases under investigation at the particular wavelengths of light emitted by these lamps. Particularly for molecules whose absorption spectra contain structure narrow compared with the slit function, the accuracy of the values of absorption coefficients and ionization quantum yields measured with a monochromator will depend on the resolution of the monochromator. It was thus best for our purposes that we determine

\footnotetext{
*Work supported in part by U.S. Atomic Energy Commission.

' Fir wres in brackets indicate the literature references at the end of this paper.
}

the values of these constants with the same lamps that are used in the photolysis experiments. Although this method gives the ionization quantum yield and absorption coefficient only at selected wavelengths (the rare gas resonance lines), they are, for those using rare gas resonance lamps as photochemical light sources, the wavelengths of primary interest. These measurements also provide a rough check of the accuracy of the values reported for these constants at these energies as determined by other methods. In the case of the neon resonance lines it is more difficult to correlate our measurements with others, since the lamp emits two resonance lines. However, these lines are rather close together and the $73.6 \mathrm{~nm}$ line is about three times the intensity of the $74.4 \mathrm{~nm}$ line [4]. Especially for those substances in which there is no structure in the absorption curve, these measurements should be good.

Since the experiments reported in this paper were performed, a somewhat similar investigation has been reported by Bennett et. al., [5] on the absorption coefficients and ionization yields of a number of compounds at $58.4 \mathrm{~nm}$. In general, there is good agreement between their results and ours.

\section{Experimental Procedure}

\subsection{Helium and Neon Resonance Lamps}

The details of constructing and filling the helium and neon resonance lamps have been given before [6]. 
The operational characteristics, such as the effect of the rare gas pressure and of the power of the microwave generator on the intensity of the lamp, have also been discussed. To summarize briefly, the lamps are enclosed glass constructions, filled with a low pressure of rare gas, fitted with thin aluminum windows (100$200 \mathrm{~nm}$ thick), and operated with a microwave generator. The helium pressure was about 1.5 torr while the neon pressure was approximately 2 torr in the two respective lamps. The intensity of these lamps $\left(\sim 10^{12}\right.$ quantum/s) was very steady, with less than a five percent fluctuation over the time necessary for one set of measurements ( 1 to $2 \mathrm{~h}$ ). Over a longer period of time there was a gradual decay in flux mainly due to a deterioration of the aluminum window. This decrease amounted to about 10 percent over a $24 \mathrm{~h}$ period.

\subsection{Double Cell Arrangement}

In order to obtain accurate values of ionization quantum yields and extinction coefficients, the double cell arrangement, shown in figure 1, was used. Each cylindrical chamber of the double cell is about $11 \mathrm{~cm}$ long and $5 \mathrm{~cm}$ in diameter and has a volume of approximately $200 \mathrm{~cm}^{3}$. The two compartments, both of which contain a set of parallel circular nickel electrodes, are separated by an aluminum window. The gas that is to be investigated is introduced into the chamber immediately adjacent to the lamp. The second chamber contains a gas that serves as a standard actinometer. In this way, the light transmitted through the material

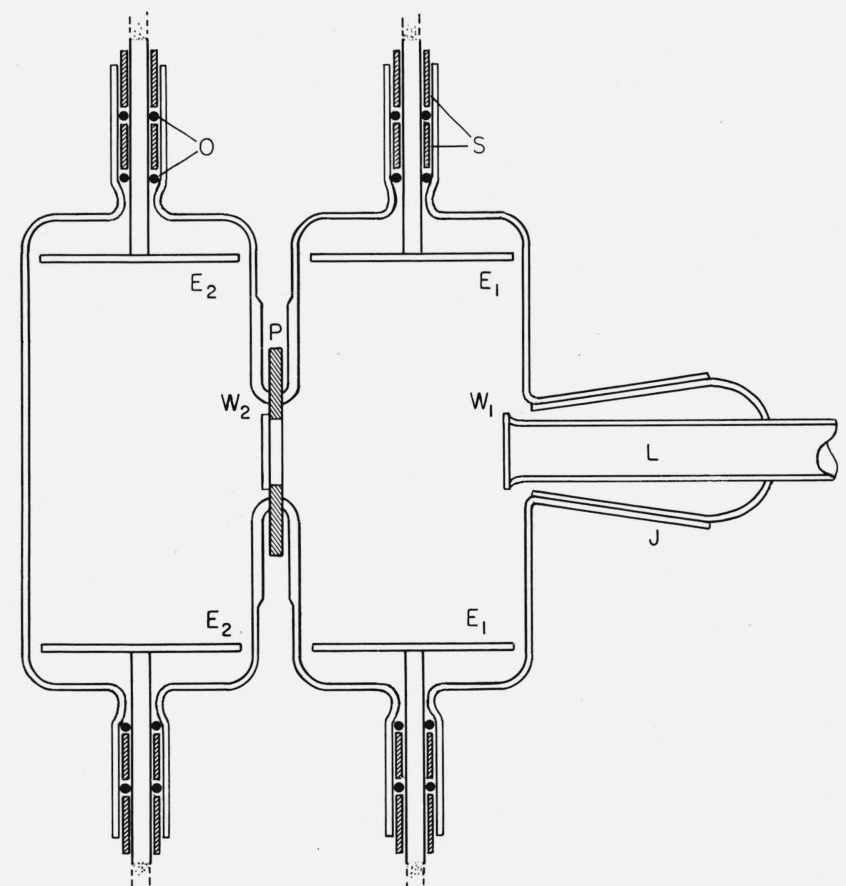

FIGURE 1. A double cell arrangement for actinometric

$\mathrm{L} \quad$ measurements
$\mathrm{E}_{1}$ and $\mathrm{E}_{2}-$ - nickel electrodes
$\mathrm{W}_{1}$ and $\mathrm{W}_{2}-$ aluminum windows
$\mathrm{P}$ - Pyrex disk.
$\mathrm{O}$ and $\mathrm{S} \quad$ - viton "O" rings and glass separators, respectively through which the inter-
$\mathrm{J} \quad$ - electrode distance is varied
- a standard type joint $34 / 45$.

in the first cell can be directly determined by a saturation current measurement in the second cell, or when the first cell is evacuated, the constancy of the intensity of the lamp can be checked.

In some cases, where the saturation ion current measurements show good plateaus, only a single cell is necessary. All of the measurements on the fluoroand chloromethanes were made in the single cell apparatus. Most of the other results were obtained in the double cell arrangement.

\subsection{Actinometry}

The standard actinometric gas can in principle be any gas for which the quantum yield of ionization at the wavelength of interest is known. In practice, experimental errors of measurement can be minimized if the actinometric gas gives a constant saturation current over a wide voltage range. This will be the case for gases which have a low absorption coefflcient at the wavelength emitted by the lamp. When saturation ion currents are measured in a gas having a high absorption coefficient at the particular wavelength of light, the saturation current which is observed is usually obtained over a very short voltage range and, therefore, is not always well characterized. The plateau of the saturation current can be improved by adding to the absorbant gas some other gas which is transparent to the radiation being used. For example, neon is transparent to the helium resonance radiation and helium is transparent to neon resonance radiation, so each of these rare gases can be used as a diluent for strongly absorbing gases irradiated with the lamp giving off the resonance lines of the other gas.

Rare gases in general are considered to have an ionization efflciency of unit when they absorb a photon of energy greater than their ionization energies [7]. However those rare gases which are ionized at the helium and neon resonance lines also absorb this light very strongly. For instance the absorption coefficients for argon at the neon and helium lines are 900 and 975 atm $^{-1} \mathrm{~cm}^{-1}$ respectively [8]. Thus the plateau of the saturation current measured in argon (fig. 2) is not well characterized. However, when helium is added to the cell with the argon, a short well defined plateau is obtained. The added helium acts as a moderator so that ion multiplication does not occur until higher applied voltages.

Hydrogen gas is used as a secondary standard because of its lower absorption coefficients (300 and 170 atm ${ }^{-1} \mathrm{~cm}^{-1}$ at 74 and $58 \mathrm{~nm}$ respectively) [9]. A very well defined plateau is obtained for the saturation ion current measured in pure hydrogen (fig. 2) over a wide pressure range. The ionization quantum yield of hydrogen is 0.94 and 1.00 at the neon and helium resonance lines respectively.

In order to obtain good saturation current plateaus, as well as meaningful absolute values for the saturation ion currents, the investigator must also be aware of the effects on the measured currents of such parameters as lamp flux and pressure of the absorbant gas. These effects have been discussed in detail previously 


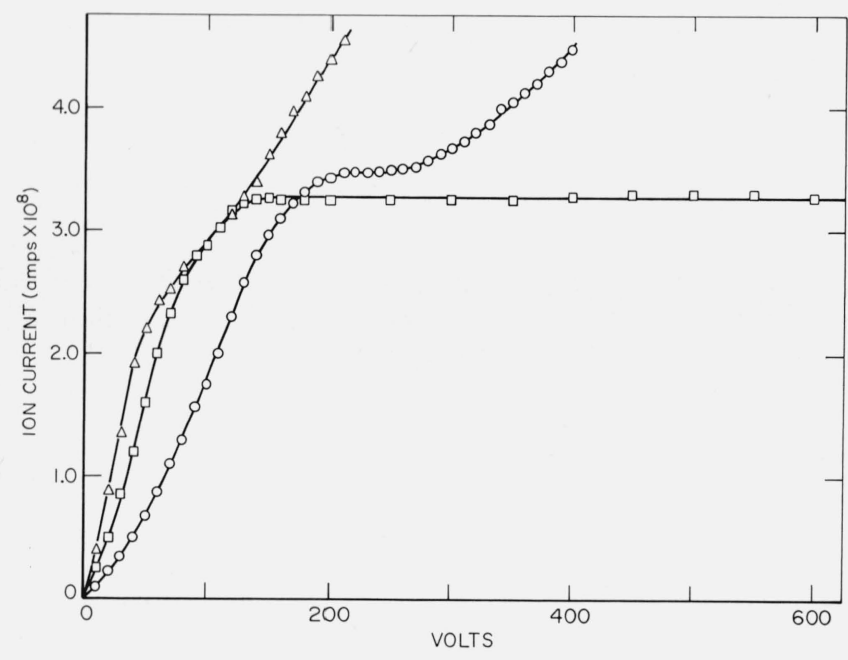

FigURE 2. A plot of ion current versus applied voltage at constant intensity of the neon resonance lamp for various substances $\square 5$ torr of hydrogen
$\triangle 0.8$ torr of argon

12 torr of a helium-argon (14:1) mixture.

[10], and thus will only be summarized here. If the number of quanta emitted per unit area of the window is too high, no saturation current can be obtained; electron multiplication occurs before the plateau of the saturation ion current is reached. It has been shown that if the pressure of the sample gas is too high, all the ions are not collected at the electrodes and the measured current is too low. Thus the investigator should take care to find the optimum conditions of flux and pressure for each individual gas, in order to obtain meaningful saturation ion current measurements.

\subsection{Materials}

Most of the chemicals used in this investigation were of research grade quality, and were used without further purification except for degassing. The nitric oxide was stored over silica gel. Most of the hydrocarbons were purified on a gas chromatograph. Methane was degassed at solid nitrogen temperature.

\section{Results and Discussion}

\subsection{Ionization Quantum Yields}

At pressures such that all the photons are absorbed quantum yields of ionization, $\Phi_{+}(x)$, are determined by comparing the saturation ion current $\left(i_{x}\right)$ obtained in gas $(x)$ for a given flux with the saturation ion current $\left(i_{\mathrm{st}}\right)$ obtained for the same flux in some standard gas whose ionization quantum yield, $\Phi_{+}(\mathrm{st})$, is known at the wavelength of interest. Thus $\Phi_{+}(x)=\Phi_{+}(\mathrm{st})$ $\left(\frac{i_{x}}{i_{\mathrm{st}}}\right)$. The ionization quantum yields determined in this laboratory for selected gases at the argon, neon and helium resonance lines are given in table 1 . The
TABLE 1. Ionization quantum yields

\begin{tabular}{|c|c|c|c|}
\hline \multicolumn{2}{|c|}{$106.7-104.8 \mathrm{~nm}^{\text {a }}$} & \multirow{3}{*}{$\begin{array}{c}74.4-73.6 \mathrm{~nm} \\
{ }^{*} 1.00(1.00)^{\mathrm{c}} \\
1.00(1.00)^{\mathrm{c}}\end{array}$} & \multirow{2}{*}{$\begin{array}{c}58.4 \mathrm{~nm} \\
* 1.00(1.00)^{\mathrm{c}}\end{array}$} \\
\hline Ar & 0.00 & & \\
\hline $\mathrm{Xe}$ & .00 & & $\begin{array}{r}\text { n.d. }(1.00)^{\mathrm{c}} \\
(1.00)^{\mathrm{e}}\end{array}$ \\
\hline $\mathrm{H}_{2}$ & .00 & 0.94 & $\begin{array}{r}1.00(1.00)^{\mathrm{e}} \\
(1.00)^{\mathrm{g}}\end{array}$ \\
\hline $\mathrm{H}_{2} \mathrm{O}$ & .00 & $0.72(0.6)^{\mathrm{d}}$ & $\begin{array}{r}\text { n.d. }(1.00)^{\mathrm{e}} \\
(0.75)^{\mathrm{d}}\end{array}$ \\
\hline $\mathrm{H}_{2} \mathrm{~S}$ & .52 & 1.03 & n.d. \\
\hline $\mathrm{O}_{2}$ & .00 & 0.91 & $\begin{array}{r}0.95(1.00)^{\mathrm{e}} \\
(1.00)^{\mathrm{h}}\end{array}$ \\
\hline $\mathrm{CO}$ & .00 & 0.81 & $\begin{array}{r}0.96(0.97)^{\mathrm{e}} \\
(0.97)^{\mathrm{i}}\end{array}$ \\
\hline $\mathrm{CO}_{2}$ & .00 & 0.87 & $\begin{array}{r}1.00(1.00)^{\mathrm{e}} \\
(0.99)^{\mathrm{i}}\end{array}$ \\
\hline $\mathrm{N}_{2}$ & .00 & 0.90 & $\begin{array}{r}0.98(1.00)^{\mathrm{e}} \\
(1.00)^{\mathrm{h}}\end{array}$ \\
\hline NO & $* .71^{\mathrm{b}}$ & 0.77 & $\begin{array}{r}0.95(0.98)^{\mathrm{e}} \\
(0.94)^{\mathrm{b}}\end{array}$ \\
\hline $\mathrm{N}_{2} \mathrm{O}$ & .00 & 0.88 & n.d. $(0.98)^{\mathrm{e}}$ \\
\hline $\mathrm{NH}_{3}$ & n.d. & $0.77(0.55)^{\mathrm{d}}$ & $\begin{array}{r}\text { n.d. }(1.00)^{\mathrm{e}} \\
(0.65)^{\mathrm{d}}\end{array}$ \\
\hline $\mathrm{CH}_{4}$ & .00 & $1.00(0.85)^{\mathrm{d}}$ & $\begin{array}{r}0.96(1.00)^{\mathrm{e}} \\
(0.75)^{\mathrm{d}}\end{array}$ \\
\hline $\mathrm{C}_{2} \mathrm{H}_{6}$ & .05 & $\begin{array}{c}1.00(0.85)^{\mathrm{d}} \\
(1.0)^{\mathrm{f}}\end{array}$ & $\begin{array}{l}(0.98)^{\mathrm{e}} \\
(1.0)^{\mathrm{f}}\end{array}$ \\
\hline $\mathrm{C}_{3} \mathrm{H}_{8}$ & .25 & $1.00(1.0)^{\mathrm{f}}$ & $(1.0)^{\mathrm{f}}$ \\
\hline$n-\mathrm{C}_{4} \mathrm{H}_{10}$ & .32 & $1.00(1.0)^{\mathrm{f}}$ & $(1.0)^{\mathrm{f}}$ \\
\hline$c-\mathrm{C}_{6} \mathrm{H}_{12}$ & .49 & 1.00 & n.d. \\
\hline $\mathrm{C}_{6} \mathrm{H}_{6}$ & $\begin{array}{l}.47 \\
80\end{array}$ & $\begin{array}{l}1.00 \\
.92(0.85)^{\mathrm{d}}\end{array}$ & $\begin{array}{l}\text { n.d. } \\
\text { n.d. }(1.0)^{d}\end{array}$ \\
\hline $\mathrm{C}_{2} \mathrm{H}_{4}$ & .20 & $\begin{array}{c}.98(0.7)^{\mathrm{d}} \\
(1.0)^{\mathrm{r}}\end{array}$ & $0.98(1.0)^{\mathrm{f}}$ \\
\hline $\mathrm{C}_{2} \mathrm{H}_{5} \mathrm{OH}$ & .20 & .92 & n.d. $(0.98)^{\mathrm{e}}$ \\
\hline $\mathrm{CH}_{3} \mathrm{COCH}_{3}$ & .21 & 1.00 & n.d. \\
\hline $\mathrm{CH}_{3} \mathrm{I}$ & .74 & 1.04 & n.d. \\
\hline $\mathrm{CF}_{+}$ & n.d. & $0.33(0.24)^{\mathrm{d}}$ & n.d. \\
\hline $\mathrm{CF}_{3} \mathrm{Cl}$ & n.d. & .89 & n.d. \\
\hline $\mathrm{CF}_{2} \mathrm{Cl}_{2}$ & n.d. & .96 & n.d. \\
\hline $\mathrm{CFCl}_{3}$ & n.d. & $\begin{array}{r}.94 \\
96\end{array}$ & n.d. \\
\hline $\mathrm{CCl}_{4}$ & n.d. & .96 & n.d. \\
\hline
\end{tabular}

*Standard.

n.d. - not determined

(References are for literature values that are given in parentheses.

ausloos, P., and Lias, S. C., Rad. Res. Rev. 1, 75 (1968).

'Watanabe, K., Matsunaga, F. M. Sakai, H., Appl. Opt. 6, 391 (1967)

c Samson, J. A. R... J. Opt. Soc. Am. 54,6 (1964).

d Vetzer, P. H.. and Cook, (, R.. J. Chem. Phys. 41, 642 (1964)

e Bennett, S. W.. Tellinghuisen, J. B., and Phillips, I.. F.. J. Phys. Chem. 75, 719 (1971).

(Sente . W. J. Chem Phys. 37, a 032 (1962).

${ }^{R}$ Cook, G. R., and Metzoer, P. H..J. Opt. Soc. Am. 54, 968 (1964)

h Huffman, R. E., Can. J. Chem. 47, 1823 (1969).

Cairns, R. B., and Samson, J. A. R., J. Geophys. Rev. 70, 99 (1965).

values reported by Bennett et al. [5], at the helium resonance line are also included in this table, as well as some earlier results obtained with windowless monochromators. In general, for the determinations of ionization quantum yields at the neon resonance lines, excess helium was added, while at the helium line, excess neon was added; as mentioned above, the plateau of the saturation ion current can be improved by adding a nonabsorbing diluent to the gas of interest. For the ionization quantum yield measurements at the argon resonance lines, the standard of comparison was the saturation ion current generated in nitric oxide. The ionization quantum yield of nitric oxide is known over this wavelength region [11]. 
In general there is very good atreement between our values and those of Bennett et al., [5] for the ionization quantum yields at the helium line. It was more difficult to obtain well characterized saturation ion current plateaus with the helium lamp than with the neon lamp. This is especially true when the extinction coefficient is high, as it is with organic compounds. Thus, these values of ionization quantum yields are not so reliable as the others given in table 1.

In general for most of the compounds which we investigated, the ejected electron does not have enough energy to cause additional ionization. Moreover the addition of an inert diluent, such as helium or neon, should moderate the energy of the electron so that secondary ionization is not important. Nevertheless, in two cases, $\mathrm{H}_{2} \mathrm{~S}$ and $\mathrm{CH}_{3} \mathrm{I}$, the ionization quantum yield is slightly above one at the neon resonance lines. At this time it is not possible to decide whether this is real, or if it is caused by unknown experimental effects. These values seem to be just outside the limits of experimental error which we estimate to be \pm 0.02 units for the neon lamp.

For the inorganic substances, especially the diatomic molecules, there is generally considerable structure in the absorption spectra; because of this, and because the neon resonance lamp has two lines at 73.6 and $74.4 \mathrm{~nm}$, a comparison between our measured values of the ionization quantum yields and values reported in the literature for this energy region is not meaningful. Our values will, to some extent, depend on the degree of reversal of the resonance lines emitted by the neon lamp. This reversal of the resonance lines depends on the pressure of neon in the lamp. However for those cases where the extinction coefficient is not changing rapidly over the region $73.6-74.4 \mathrm{~nm}$, a comparison can be made. This is particularly true for the organic compounds. In general our results agree reasonably well with the results of Schoen [13] but are somewhat higher than the results of Metzger and Cook $[9,12]$.

The saturation-ion current measurements can also be used to obtain the absolute intensity of light entering the cell, if the ionization efficiency, $\Phi_{+}(x)$, for a compound, $x$, is known and if there is complete absorption of the light by the compound. Thus $Q_{a}=6.24 \times 10^{18}\left(\frac{i_{x}}{\Phi_{+}}\right)$where $Q_{a}$ is the absolute flux of the absorbed light in quanta $\mathrm{s}^{-1}$ and $i_{x}$ is the saturation ion current of $x$ in amperes.

\subsection{Extinction Coefficients}

The determination of extinction coefficients using the double chamber cell is quite straightforward. The liwht transmitted through the evacuated cell $\left(T_{0}\right)$ and the light transmitted through the absorbing material $(T)$ at a given pressure are simply proportional to the saturation ion currents, $I_{0}$ (saturation ion current in second cell with the first cell evacuated) and $I$ (saturation ion current in second cell with pressure, $p$, in the first cell). Using Beer's Law, we write

$$
\frac{I}{I_{o}}=e^{-\epsilon x p}
$$

where $x$ is the path length of the first cell in $\mathrm{cm}, p$ is the pressure in atm, and $\epsilon$ is the extinction coefficient in $\mathrm{cm}^{-1} \mathrm{~atm}^{-1}$. The path length in the first cell was $3.7 \mathrm{~cm}$.

The extinction coefficients can also be determined from the saturation ion current measurements made in the first cell or in a single cell with the absorbing material. In this case the incident flux and the absorbed flux at a given pressure are simply proportional to the saturation ion current $I_{o}^{\prime}$ (saturation ion current for the total absorption of light) and $I_{a}^{\prime}$ (saturation ion current with pressure, $p$ ), measured in the first cell. Thus, we again have from Beer's Law:

$$
\frac{I_{o}^{\prime}-I_{a}^{\prime}}{I_{o}^{\prime}}=e^{-\epsilon x p^{\prime}}
$$

In the single cell apparatus the path length was 5.0 $\mathrm{cm}$.

In figure 3 are plotted $\log I / I_{0}$ versus pressure for propane irradiated with the neon lamp in the double cell. Also included on this plot are the calculations made from saturation ion current measurements in the first cell only. The agreement between the two sets of measurements is good over the range where well characterized saturation ion current plateaus are obtained in the first cell. As mentioned above, these measurements were obtained with helium added to the propane. This addition of helium, besides enhancing the plateau of the saturation ion current measurements in the first cell, also improved the accuracy of the pressure readings. The ratio of helium to propane was 24 .

Figure 4 shows the plot of log $I / I_{0}$ versus pressure for propane irradiated with the helium lamp in the double cell. In this case neon was added to the propane as a diluent. The ratio of neon to propane was 24 .

\begin{tabular}{|c|c|c|c|c|}
\hline \multirow[b]{2}{*}{$\begin{array}{l}\mathrm{H}_{2} \\
\mathrm{CO}_{2} \\
\mathrm{C}_{3} \mathrm{H}_{8} \\
\mathrm{CF}_{4} \\
\mathrm{CF}_{3} \mathrm{Cl} \\
\mathrm{CF}_{2} \mathrm{Cl}_{2} \\
\mathrm{CFCl}_{3} \\
\mathrm{CCl}_{4}\end{array}$} & \multicolumn{2}{|c|}{$\frac{74.4-73.6 \mathrm{~nm}}{\left(\mathrm{~cm}^{-1} \mathrm{~atm}^{-1}\right)}$} & \multicolumn{2}{|c|}{$\frac{58.4 \mathrm{~nm}}{\left(\mathrm{~cm}^{-1} \mathrm{~atm}^{-1}\right)}$} \\
\hline & $\begin{array}{r}300 \\
585 \\
2950 \\
\text { d } 1370 \\
\text { d } 2270 \\
\text { d } 3150 \\
\text { d } 4520 \\
\text { d } 5300\end{array}$ & $\begin{array}{l}\text { a }(3000) \\
\text { b }(1050)\end{array}$ & $\begin{array}{l}{ }^{\mathrm{d}} 185 \\
\text { n.d. } \\
2240 \\
\text { n.d. } \\
\text { n.d. } \\
\text { n.d. } \\
\text { n.d. } \\
\text { n.d. }\end{array}$ & b, c (175) \\
\hline
\end{tabular}

TABLE 2. Extinction coefficients

(References are for literature values that are given in parenthesis.)

a Schoen, R. I., J. Chem. Phys. 37, 2032 (1962).

b Cook, G. R., Metzger, P. H., Ogawa, M., Becker, R. A., and Ching, B. K., Aerospace Corp. Report No. TDR-469(9260-01)-4.

'Bennett, S. W., Tellinghuisen, J. B., Phillips, L. F., J. Phys. Chem. 75, 719 (1971).

d These results are from the single cell.

n.d. - not determined. 


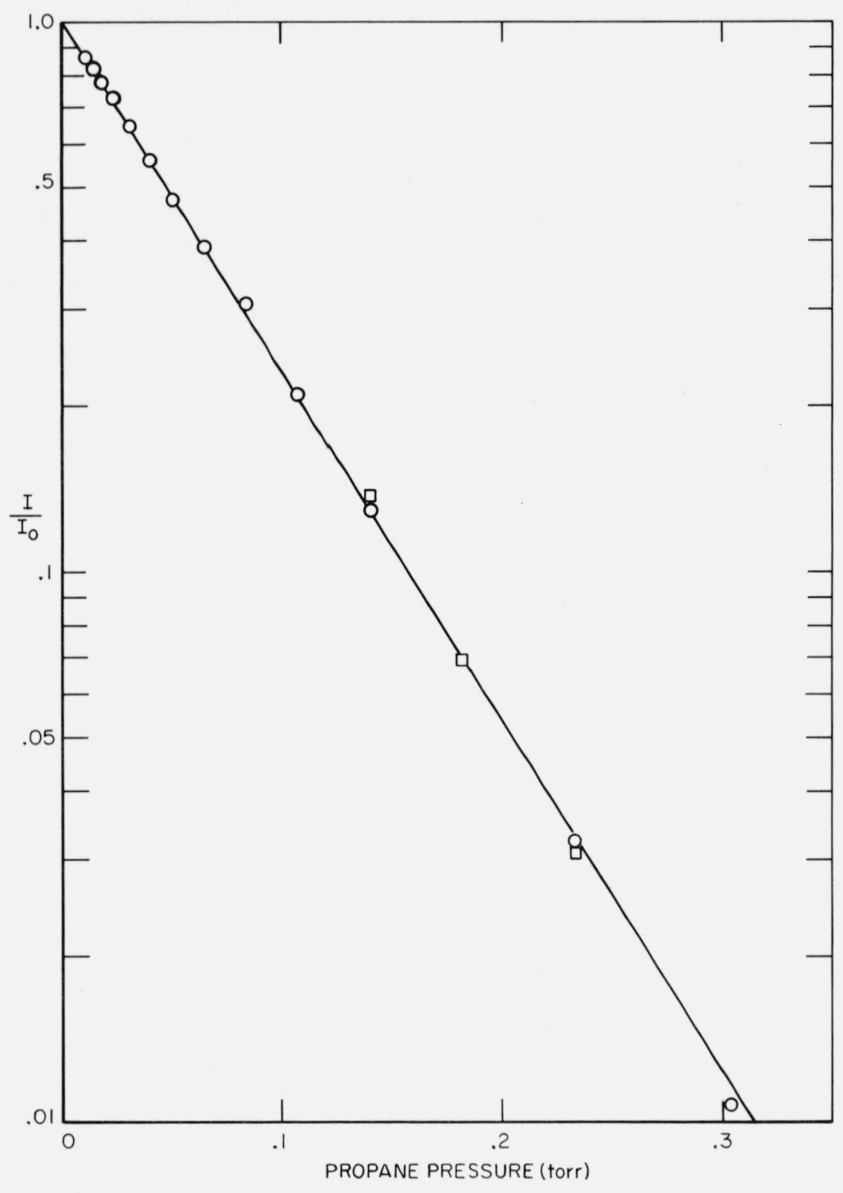

FiguRE 3. A plot of $\mathrm{I} / \mathrm{I}_{0}$ versus propane pressure for the neon resonance lamp

0 . Measurements of $I / I_{0}$ made in second cell for neon lamp. 0 . Measurements of $\left(I_{0}^{\prime}-I_{0}^{\prime}\right) / I_{0}^{\prime}$ made in first cell for neon lamp.

Similar good Beer's Law plots were obtained for the other gases listed in table 2, independent of whether the double cell or single cell arrangement was used. The single cell apparatus did necessitate using higher ratios of helium to the gas of interest in order to obtain good plateaus for those substances with higher extinction coefficients. For carbon tetrachloride which had the highest extinction coefficient, the ratio of $\mathrm{He} / \mathrm{CCl}_{4}$ was 76 . We estimate our experimental error for the extinction coefficients to be about 5 percent.

\section{References}

[1] Schoen, R. I., Can. J. Chem. 47, 1879 (1969).

[2] de Reilhac, L., Damany-Astoin, N. and Romand, J., Spectrochimica Acta 25A, 19 (1969).

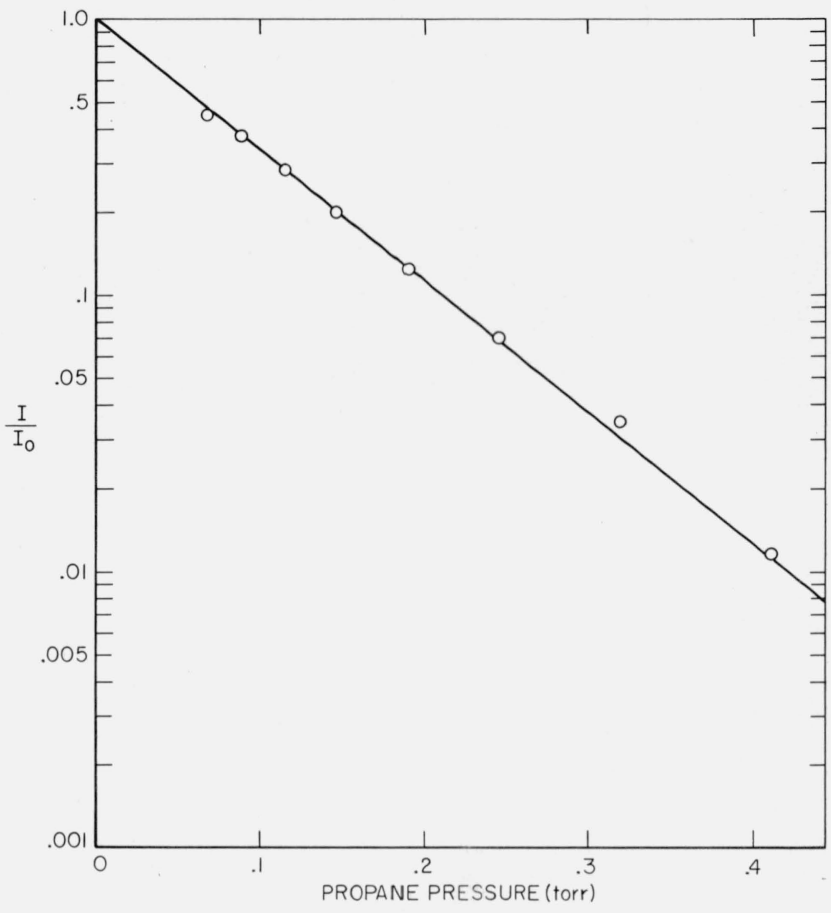

Figure 4. A plot of $\mathrm{I} / \mathrm{I}_{0}$ versus propane pressure for the helium resonance lamp using the double cell arrangement.

[3] Rebbert, R. E., and Ausloos, P., J. Am. Chem. Soc. 90, 7370 (1968); Lias, S. G., Rebbert, R. E., and Ausloos, P. J. Chem. Phys. 52, 773 (1970).

[4] Boyce, J. C., Phys. Rev. 46, 378 (1934); Samson, J. A. R., Techniques of Vacuum Ultraviolet Spectroscopy, Chapter 5, (John Wiley and Sons, Inc., New York, 1967).

[5] Bennett, S. W., Tellinghuisen, J. B., and Phillips, L. F., J. Phys. Chem. 75, 719(1971).

[6] Gorden, R. Jr., Rebbert, R. E., and Ausloos, P., Nat. Bur. Stand. (U.S.), Tech. Note 496, 55 pages (Oct. 1969).

[7] Samson, J. A. R., J. Opt. Soc. Am. 54, 6 (1964).

[8] Samson, J. A. R., Adv. Atomic and Molecular Physics, Vol. II (Academic Press, New York, 1966).

[9] Cook, G. R., and Metzger, P. H., J. Opt. Soc. Am. 54, 968 (1964).

[10] Gorden, R. Jr., Doepker, R., and Ausloos, P., J. Chem. Phys. 44, 3733 (1966); Ausloos, P., and Lias, S. G., Rad. Res. Rev. 1, 75 (1968).

[11] Watanabe, K., Matsunaga, F. M., and Sakai, H., Appl. Opt. 6, 391 (1967).

[12] Metzger, P. H., and Cook, G. R., J. Chem. Phys. 41, 642 (1964).

[13] Schoen, R. I., J. Chem. Phys. 37,2032 (1962).

(Paper 75A5-681) 